\title{
Berücksichtigung von Genderaspekten in den neuen gestuften Studiengängen in der Raumplanung ${ }^{1}$
}

\author{
Considering aspects of gender within \\ the new tiered degree programs in spatial \\ planning
}

Keywords: Gleichstellung, Gender Mainstreaming, Akkreditierung, Raumplanungsstudiengänge in Deutschland, Innovationsbedarf in der Ausbildung

Keywords: gender equality, gender mainstreaming, accreditation, degree programs of spatial planning in Germany, requirement of innovation in (higher) education

\section{Kurzfassung}

Der Beitrag untersucht, inwieweit die im Rahmen des sog. „Bologna-Prozesses“ entstandenen gestuften Raumplanungsstudiengänge in Deutschland Gender-Inhalte und -Module in ihre Studienprogramme aufgenommen haben. Einbezogen in diesen Quervergleich wurden die Hochschulen in Cottbus, Hamburg, Berlin, Dortmund und Kaiserslautern.

Die Autorin stellt fest, dass die vorhandenen Traditionen im Einzelfall fortgesetzt, wo diese jedoch nicht vorhanden waren, auch nicht eingeführt wurden. Abschließend weist sie darauf hin, dass eine zukunftsfähige Raumentwicklung ohne Gleichstellungsorientierung nicht denkbar ist, und rät den Hochschulen, diesen Bedarf bei der Weiterentwicklung ihrer Studiengänge zu berücksichtigen.

\section{Abstract}

The article analyses in how far the new tiered degree programmes of spatial planning, which have been developed within the so-called „Bologna Process“, are containing any gender contents or modules. This examination includes the German universities of Cottbus, Hamburg, Berlin, Dortmund and Kaiserslautern.

The author points out that gender traditions are proceeded where they already have been existent. However; if those traditions have not been existent before, universities did not establish any gender aspects in the course of the reforms. Finally, it is suggested that sustainable spatial development has to be supported by gender aspects. Therefore, universities are advised to further develop those aspects within their study programme.

Am 19. Juni 1999 haben die Europäischen Bildungsministerinnen und -minister in Bologna eine gemeinsame Erklärung zur Schaffung eines gemeinsamen Hochschulraumes verabschiedet. In der so genannten „Bologna-Erklärung“ verpflichteten sich die beteiligten Staaten, bis 2010 folgende Ziele zu erreichen:
- Entwicklung eines wettbewerbsfähigen und dynamischen Hochschul- und Forschungsraumes,

- Transparenz und Vergleichbarkeit im Interesse einer europaweiten Mobilität von Lernenden und Lehrenden,

- Einführung von Bachelor- und Masterstudiengängen mit einem transparenten Leistungspunktesystem. 
Seither wird in allen europäischen Ländern an der Konzeption der neuen Studiengänge gearbeitet. Der erste gestufte Raumplanungsstudiengang in Deutschland wurde im Juni 2005 an der Brandenburgischen Technischen Universität (BTU) Cottbus eingeführt, die HafenCity Universität (HCU) Hamburg folgte im Oktober 2005, die Technische Universität Berlin im Februar/Juli 2006. An der Technischen Universität Kaiserslautern und an der Technischen Universität Dortmund wurden die neuen Bachelorstudiengänge in Raumplanung zum Wintersemester 2007/08 gestartet. $^{2}$

Geschlechtergerechtigkeit war in der Bologna-Erklärung von 1999 kein Thema. Der deutsche Wissenschaftsrat hatte am 15.5.1998 zwar bereits seine „Empfehlungen zur Chancengleichheit von Frauen in Wissenschaft und Forschung "verabschiedet, diese bezogen sich jedoch auf die personelle Gleichstellung in den Hochschulstrukturen, nicht auf Lehrziele und Forschungsinhalte. Ebenso wenig führten diese Empfehlungen dazu, Gleichstellungsaspekte in die Grundlagen des entstehenden europäischen Hochschulraums zu integrieren.

\section{Der Amsterdamer Vertrag 1999}

Mit dem Inkrafttreten des Amsterdamer Vertrages am 01.05.1999 hatten sich jedoch kurz zuvor die rechtlichen Rahmenbedingungen geändert: Durch die Formulierungen in Art. 2 und 3 Abs. 2 dieses Vertrages war Gender Mainstreaming verbindlich als Leitprinzip für alle politischen Entscheidungen und Maßnahmen in der EU festgeschrieben worden. Diese Normen mussten jedoch erst von den Mitgliedsstaaten eingeführt werden, um das politische Handeln auch für die nationalen Politiken entsprechend anzupassen.

\begin{abstract}
Amsterdamer Vertrag vom 1. Mai 1999
Art. 2: „Aufgabe der Gemeinschaft ist es, durch die Errichtung eines Gemeinsamen Marktes und einer Wirtschafts- und Währungsunion sowie durch die Durchführung der in den Artikeln 3 und 4 genannten gemeinsamen Politiken und Maßnahmen in der ganzen Gemeinschaft (...) die Gleichstellung von Männern und Frauen (...) zu fördern."

Art. 3 Abs. 2: „Bei allen in diesem Artikel genannten Tätigkeiten wirkt die Gemeinschaft darauf hin, Ungleichheiten zu beseitigen und die Gleichstellung von Männern und Frauen zu fördern."
\end{abstract}

Die deutsche Bundesregierung hat auf Grundlage des im Amsterdamer Vertrag festgelegten Staatsziels die Gleichstellung von Frauen und Männern als durchgängiges Leitprinzip ihres Handelns mit Kabinettbeschluss vom 23.6.1999 anerkannt und beschlossen, diese mittels der Strategie des Gender Mainstreaming zu fördern. In Ausführung dieses Beschlusses wurde am 26.7.2000 in $\$ 2$ der Gemeinsamen Geschäftsordnung der Bundesministerien die Verpflichtung aller Ressorts festgelegt, diesen Ansatz bei allen politischen, Norm gebenden und verwaltenden Maßnahmen der Bundesregierung $\mathrm{zu}$ beachten. Im Bundesgleichstellungsgesetz ist der Grundsatz des Gender Mainstreaming in $\$ 2$ verankert. ${ }^{3}$

\section{Konsequenzen für den Bologna-Prozess}

Seit der Verabschiedung der Bologna-Erklärung finden alle zwei Jahre Konferenzen der europäischen Hochschulministerinnen und -minister statt, um den Stand der Umsetzung zu überprüfen und gemeinsam zu diskutieren. An der Konferenz vom 19.9.2003 wurde im so genannten „Berliner Kommuniqué“ erstmals auch das Ziel der Geschlechtergerechtigkeit festgeschrieben:

„Die Ministerinnen und Minister bekräftigen erneut die Bedeutung der sozialen Dimension des Bologna-Prozesses. Die Notwendigkeit, die Wettbewerbsfähigkeit zu verbessern, muss mit dem Ziel, der sozialen Dimension des Europäischen Hochschulraumes größere Bedeutung zu geben, in Einklang gebracht werden; dabei geht es um die Stärkung des sozialen Zusammenhalts sowie den Abbau sozialer und geschlechtsspezifischer Ungleichheit auf nationaler und europäischer Ebene“.

(vgl. http://www.bologna-berlin2003.de/pdf/Communique1. $p d f)$

Die Umsetzung des Bologna-Prozesses und die damit verbundenen Konsequenzen für die Gleichstellung von Frauen und Männern und dle Frauen- und Geschlechterforschung wurden in der Folge auf verschiedenen Tagungen diskutiert. Die Bundeskonferenz der Frauenund Gleichstellungsbeauftragten an Hochschulen (BuKoF) thematisierte anlässlich ihrer Jahrestagung 2003 die Auswirkungen der künftigen Studienabschlüsse auf die Bildungs- und Qualifikationschancen von Frauen sowie die Auswirkungen auf die Frauenund Geschlechterforschung und die Einbeziehung von Genderaspekten in die Akkreditierungsverfahren (vgl. Löther 2004; Becker/Jansen-Schulz/Kortendiek/ Schäfer 2006). An der Universität Siegen fand im Oktober 2003 die Tagung „Frauen im Aufwind des BolognaProzesses. Analysen und Handlungsempfehlungen zur Herstellung von Chancengleichheit bei der Einführung von Bachelor-/Masterstudiengängen an deutschen Hochschulen" statt. ${ }^{4}$ Für die geschlechtergerechte Umstellung der Studiengänge wurden dabei folgende Postulate entwickelt:

- Integration der Frauen- und Geschlechterforschung in Forschung und Lehre,

- Gestaltung der Inhalte, Lehrstrukturen und Lehrformen so, dass Studierende beiderlei Geschlechts gleichermaßen angesprochen werden, 
- Stärkung der Genderkompetenz aller am Studienreformprozess Beteiligten.

Im Rahmen der Eckpunkte für die Weiterentwicklung der Akkreditierung in Deutschland hat die Kultusministerkonferenz vom 15.10.2004 beschlossen: „Außerdem (...) stellt der Akkreditierungsrat sicher, dass der Gender-Mainstreaming-Ansatz des Amsterdamer Vertrages (...) sowie die entsprechenden nationalen Regelungen im Akkreditierungssystem berücksichtigt und umgesetzt werden" (Kultusministerkonferenz 2004: 6).

Im Gesetz zur Errichtung der Stiftung zur Akkreditierung der Studiengänge in Deutschland vom 15.2.2005 wurde im Weiteren verankert, dass „die Organe (...) bei allen Vorschlägen und Entscheidungen die geschlechtsspezifischen Auswirkungen beachten" müssen (Gender Mainstreaming) ${ }^{5}$.

Inwiefern diese Vorgaben in den laufenden Verfahren umgesetzt wurden, soll im Folgenden am Beispiel der ersten akkreditierten Raumplanungsstudiengänge in Deutschland beleuchtet werden.

\section{Zur Akkreditierung der Studiengänge in Stadt- und Raumplanung in Deutschland}

Der Akkreditierungsverbund für Studiengänge der Architektur und Planung (ASAP) hat „Fachliche Standards für die Akkreditierung von Studiengängen der Stadtplanung/Raumplanung" herausgegeben (ASAP 2008). Diese enthalten jedoch weder in den Leitlinien noch in den Ausbildungszielen und -inhalten Hinweise auf die Berücksichtigung von Genderaspekten und -dimensionen in Forschung und Lehre. Dies mag u.a. auch auf die Zusammensetzung der beteiligten Gremien zurückzuführen sein (von den insgesamt elf Mitgliedern des Fachausschusses Stadt-/Raumplanung ist gerade eine Frau), hat jedoch gleichzeitig weitreichende Konsequenzen für die Integration der Frauen- und Geschlechterforschung in Lehrinhalte und Lernstrukturen.

So bleibt es vor diesem Hintergrund Sache der einzelnen Studienkommissionen, Inhalte und Erkenntnisse der Frauen- und Geschlechterforschung in die neuen Studienpläne zu integrieren. Dies dürfte jedoch - schon aufgrund der allgemeinen Unterrepräsentanz von Frauen in den höheren akademischen Gruppen der Hochschullehrerinnen und -lehrer wie des Wissenschaftlichen Mittelbaus - im Normalfall schwierig sein. ${ }^{6}$

Auch die Akkreditierungskommissionen werden nicht ausdrücklich dazu ermuntert, entsprechende Lehrinhalte einzufordern. Dazu kommt, dass Frauen hier ebenfalls unterrepräsentiert sind: In den deutschen Akkreditierungskommissionen ist derzeit im Durchschnitt nur jedes achte Mitglied eine Frau (vgl. Bührmann 2006).

Umso beachtlicher ist es, dass wenigstens zwei der neu konzipierten Studiengänge Gender-Module bzw. -Aspekte in ihre Studienpläne integriert haben.

\section{Die neuen Planungsstudiengänge in Deutschland $^{7}$}

Die neuen gestuften Planungsstudiengänge an den Hochschulen in Cottbus, Hamburg, Berlin, Dortmund und Kaiserslautern wurden in den Jahren von 2005 bis 2007 eingeführt. Von diesen fünf Studiengängen weisen nur die Studiengänge in Berlin und Dortmund überhaupt Gender-Aspekte auf. ${ }^{8}$

Im Folgenden werden die bisherigen Konzeptionen der neuen Raumplanungsstudiengänge in Deutschland einer Prüfung im Hinblick auf die bisherige Integration von Gender-Inhalten unterzogen.

\section{Cottbus}

Die Dokumente der BTU Cottbus zu den neuen Studiengängen in Stadt- und Regionalplanung sind schon sprachlich, und zwar ohne Ausnahme, nur auf (männliche) Planer fokussiert. So heißt es sowohl im Vorspann zur Beschreibung des Bachelor- wie auch des Masterstudienganges:

- „Die Aufgabe von Stadt- und Regionalplanern ist es (...)“

- „Ziel (...) ist es, einen Planer auszubilden, der (...)“

- „Das Berufsfeld des Absolventen (...) erstreckt sich (...)“

Mit den Zitaten von Alexander Mitscherlich (BA) und Max Frisch (MA) werden automatisch männliche Persönlichkeiten als Vorbilder transportiert, auch wenn es sich hierbei um einen stadt- und gesellschaftskritischen Soziologen und einen Architekten handelt, der sich zum politisch reflektierenden Schriftsteller weiterentwickelt hat.

„Um unsere Städte anders wachsen zu lassen, als es jetzt geschieht, müssten wir uns erst wieder für sie verantwortlich, von ihnen angesprochen fühlen. Die Städte aber werden nicht ansprechender werden, bevor wir nicht über sie mit Leidenschaft nachgedacht haben."

(Alexander Mitscherlich)

„Nicht die Sache selbst soll geplant werden, nur der Rahmen.“ (Max Frisch) 
Das Lernfeld reicht von der Gestaltung des einzelnen Gebäudes bis zur Stadt und ihrer Region und ist damit der am stärksten architektonisch fundierte unter den hier betrachteten fünf Studiengängen.

Weder im Bachelor noch im Master werden irgendwelche Hinweise auf Gender-Inhalte oder -Aspekte gegeben, auch wenn solche insbesondere in den Modulbereichen "Geschichte und Theorie" oder „Stadt 1“ und "Stadt 2 " sowie in den Projekten, die die inhaltliche und organisatorische Grundstruktur des Studiums bilden, denkbar wären. Möglichkeiten der Integration bieten zum Beispiel Pflicht- oder Wahlpflichtelemente wie Stadt- und Bau- bzw. Kunstgeschichte, Theorie der Architektur, Vermittlung von Planung, Gebäudekunde und Stadtplanung/Planungsmethoden und -verfahren, rechtliche Grundlagen des Planens und Bauens, Stadtmanagement, Stadt-, Regional- und Wohnsoziologie, Landschaftspflege und Dorfentwicklung, Stadterneuerung/sozial integrierte Stadt.

\section{Hamburg}

Die Studiengänge „Stadtplanung“ (BA) und „Stadtplanung und Stadtentwicklung" (MA) präsentieren sich insgesamt besonders werbewirksam. Sie werden durchgehend zweisprachig beworben, sind attraktiv aufbereitet und wirken besonders zeitgemäß und innovativ. Umso erstaunlicher ist es, dass Gender-Inhalte hier völlig fehlen. Auch wird in den „Informationen für Studienbewerber" durchgehend keine geschlechterdifferenzierte Sprache verwendet, Ausnahmen bilden nur zwei Formulierungen:

- „Der Masterstudiengang ,Stadtplanung und Stadtentwicklung` ist als aufbauender Studiengang für Absolventinnen und Absolventen des Bachelor-Studienganges ,Stadtplanung' ausgestaltet." und

- „Für die Ausbildung der Doktorandinnen und Doktoranden betreibt die HafenCity Universität eine ,Research School'."

Überzeugen aus einer frauen- und genderorientierten Perspektive könnte jedoch die Betrachtung des Studienorts als Labor: „Hier liegen die Zukunftsthemen der Stadtplanung vor der Haustür“. Die prioritäre Zuwendung zu den im wahrsten Sinne des Wortes naheliegenden Aufgabenfeldern ist spezifisch für einen frauenbezogenen Planungsansatz. Auch die Arbeit in Studienprojekten von zehn bis zwölf Studierenden, die als „Kernstück der Ausbildung“ bezeichnet wird, kommt den Anforderungen von Frauen bzw. der erfolgreichen Vermittlung sozialer Kompetenzen prinzipiell entgegen.
Ob das „Studium Fundamentale“, das im Bachelor ab dem 4. Semester angeboten wird und sich im Master über alle vier Semester erstreckt, die gewünschte Breite zu garantieren vermag, wird die Praxis zeigen - es weckt zumindest entsprechende Erwartungen: im Bachelor mit Exkursion und zusätzlichen Wahlfächern, im Master mit Elementen zu Methoden und Diskussion, Rhetorik, Business Planning, Karrieremanagement und Humanities (Soft skills).

Ziel des Bachelorstudiums ist die Vermittlung der Fähigkeit, planungsbezogene ökonomische, soziale und ökologische Prozesse sowie deren räumliche Wechselwirkungen $\mathrm{zu}$ analysieren und die Ergebnisse dieser Analysen in Handlungskonzepte umzusetzen (die Analyse und Berücksichtigung sozialer Räume wird nicht ausdrücklich erwähnt), im Weiteren die Entwicklung der Persönlichkeit und kommunikativer Fähigkeiten. Auch hier stellt sich die Frage, inwieweit im Zuge der Umsetzung auf die Unterschiede der Studierenden eingegangen werden wird, wenn eine entsprechende Differenzierung in den zugrundeliegenden Konzepten nicht vorgedacht ist.

Die neuen Studiengänge wirken insgesamt gut und übersichtlich strukturiert. Der Bachelor umfasst im Einzelnen: inhaltliche Grundlagen, methodisches Instrumentarium, systematische Orientierung, anwendungsbezogenes Fach- und Methodenwissen, fachliche Zusammenhänge, konzeptionelles Arbeiten in Gruppen und Studium Fundamentale - hier wäre viel Platz für Gender-Aspekte und entsprechend innovative fachliche Perspektiven. Der Master ist in drei Bereiche gegliedert: Formulierung, Analyse und Lösung komplexer Aufgaben; Vertiefungsmöglichkeiten, und zwar: Raum- und Infrastrukturplanung, Stadt und Gesellschaft, Projekt und Immobilie, Entwurf und Studium Fundamentale. Es stellt sich hier die Frage, warum "Gesellschaft", bzw. soziale Aspekte, als eigentliche Querschnittsthematik nur in der einen Vertiefung berücksichtigt sind.

Alles in allem sind die neuen Studiengänge an der HCU querschnitts- und praxisorientiert - Gender Aspekte könnten in diese Struktur als Querschnittsthema rein theoretisch mühelos integriert werden.

\section{Kaiserslautern}

Das umfassendste Programm eines Bachelor in Raumplanung hat die TU Kaiserslautern aufzuweisen. Das Lernfeld reicht von den Grundlagen des architektonischen Entwurfs über Grünordnungspläne, Schallschutz und Luftreinhaltung als Bestandteile der örtlichen Gesamtplanung bis hin zur überörtlichen 
Gesamtplanung und zu internationalen Aspekten. An Rahmenbedingungen der räumlichen Gesamtplanung werden Recht, Ökonomie, Soziologie (im Umfang von sogar 8 Credit Points (CP), aber nur im 1./2. Semester), Ökologie und Infrastruktur angeboten, im Bereich der Schlüsselkompetenzen Rede, Vortrag, Präsentation; Medien- und Öffentlichkeitsarbeit, Moderation und Mediation, CAD und GIS. Trotz dieses beachtlichen Umfangs an Studieninhalten sind keine Hinweise auf Gender Inhalte oder Aspekte zu finden, die Größe der Übungsgruppen von 35 bis 40 Studierenden lässt eine individuelle Vertiefung von Lehr- und Lerninhalten kaum zu.

Gender-Aspekte wären im Prinzip an vielen Stellen des Modulkatalogs integrierbar, so zum Beispiel im Bereich nachhaltige Entwicklung (der jedoch nur im Wahlpflichtmodul „Internationale Aspekte der Raumplanung" auftaucht), demographischer Wandel (dem Modul "Überörtliche Gesamtplanung“ zugeordnet) oder in Soziologie (z.B. im Zusammenhang mit „sozialen Ungleichheitsstrukturen“). Die tatsächliche Integration bleibt den einzelnen Lehrpersonen überlassen; derzeit wird dieser Aspekt in der Lehre von der Juniorprofessur für Stadt- und Regionalsoziologie vertreten.

Die Berücksichtigung einer geschlechtergerechten Sprache lässt sich anhand der vorliegenden Unterlagen (Modulhandbuch) nicht erkennen.

\section{Berlin}

An der TU Berlin existiert seit den 1970er Jahren eine fast ununterbrochene Tradition, Frauenforschung und Gender Planning in die Studieninhalte zu integrieren. Diese wurde in den 1970er Jahren von den ersten Assistentinnen im Wissenschaftlichen Mittelbau eingeführt und in den 1980er/90er Jahren von den nachwachsenden Generationen an Studentinnen und Studenten sowie Mitarbeiterinnen und Mitarbeiter fortgesetzt (vgl. Dörhöfer/Flecken/Zibell 1998).

Eins der fünf grundlegenden Studienziele ist gem. $\$ 3$ der Studienordnung für den Bachelor vom 14.2.2006 neben Analysefähigkeit, selbstverantwortlichem Lernen und Weiterbilden, wissenschaftlicher Kompetenz und Darstellungsfähigkeit „die Fähigkeit, alle Themenfelder des Studiengangs unter Genderaspekten zu bearbeiten". Gender ist hier und insgesamt an der TU Berlin $^{9}$ als Querschnittsthema eingeführt.

Allerdings findet sich gem. $\$ 6$ (6) ad. 2 StuO BA 2006 auch unter den sog. "querschnittsbezogenen Modulen“, die der Vermittlung grundlegender stadtplanerischer Fähigkeiten dienen, jedoch nicht sektoral vermittelt werden können, kein Pflichtmodul „Gender“.
Die querschnittsorientierten Module beschränken sich auf „ingenieur- und rechtswissenschaftliche Grundlagen (...)" sowie „Städtebauliches Entwerfen“.

Auffallend an den neuen Berliner Studienordnungen ist jedoch die durchgehend sprachliche Gleichbehandlung von Frauen und Männern, die gleichzeitig den Wechsel in der Reihenfolge männlicher bzw. weiblicher Bezeichnungen einschließt. So heißt es zum Beispiel in $\S 5$ (1) Nr. 1 StuO BA 2006: „einen Dozenten oder eine Dozentin“, „Tutorin oder Tutor“, „Projektbetreuer oder Projektbetreuerin" usw.

Was die tatsächliche Vermittlung von Gender-Inhalten in den Studiengängen angeht, bleibt diese insgesamt aber abhängig von entsprechend sensibilierten Lehrpersonen. Im modellhaften Studienplan für den Bachelor ist ein Element "Gender Planning“ im 4. und 6. Semester enthalten, für den Master liegen keine gleichwertigen Studienpläne vor.

\section{Dortmund}

Die bisher überzeugendste Version, auch hinsichtlich der Integration von Gender-Elementen, liegt mit der neuen Studiengangsstruktur der TU Dortmund vor. Wie an der TU Berlin kann auch hier auf eine langjährige Tradition der Frauen- und Geschlechterforschung in der Lehre zurückgeschaut werden; diese ist hier jedoch zusätzlich in den Fakultätsstrukturen verankert. Bereits 1993 wurde mit der Schaffung der Professur für Frauenforschung und Wohnungswesen die erste - und bis heute einzige - Frauenforschungsprofessur an einer bundesdeutschen Raumplanungsfakultät eingerichtet. Die Verwendung einer geschlechtergerechten Sprache ist durchgängiges Prinzip in den Unterlagen zu den neuen Studiengängen.

Dortmund hat sich als einzige Raumplanungsfakultät für einen achtsemestrigen Bachelor entschieden, an den sich ein zweisemestriger konsekutiver Master anschließt. Daneben wird der seit mehr als 20 Jahren erfolgreiche Studiengang SPRING (Spatial Planning for Regions in Growing Economies) als viersemestriger Master weitergeführt.

Argumente für den achtsemestrigen Bachelor waren zum einen der Wunsch, den interdisziplinären Charakter des bisherigen Diplomstudiengangs zu bewahren, zum anderen die Intention, forschendes Lernen zu gewährleisten: Das Absolvieren von zwei je zweisemestrigen Projekten sowie drei je einsemestrigen städtebaulichen Entwürfen entsprechen gemäß Absolventen/ -innenbefragungen den Kernelementen der beruflichen Praxis und sind das „Markenzeichen der Dortmunder Raumplanungsausbildung“, gleichzeitig Voraussetzung 
für die Eintragung in die Stadtplanerliste der Architektenkammer Nordrhein-Westfalen (Fleischhauer/ Greiwe/Scholz 2007: 174).

Ein weiteres Markenzeichen der neuen Dortmunder Raumplanungsausbildung ist ebenso die Verankerung der Geschlechterperspektive in das Curriculum, die über die Bestandteile

- „Raum und Geschlecht“ (Pflichtelement in BAModul 4 „Grundlagen der Raumplanung II Gesellschaft") und

- "Gender Planning“ (Wahlmöglichkeit in BA-Modul 16 „Planungstheorie und Planungsprozesse“)

realisiert wurde. Im Master können weitere Gender Inhalte im Wahlpflichtbereich angeboten werden, so zum Beispiel in Form einer Studienarbeit „Gesellschaftliche Differenzierung und zielgruppenorientierte Planung“ im Wahlpflichtmodul 2 „Allgemeine Planungstheorie“.

$\mathrm{Zu}$ den weiteren Besonderheiten des Dortmunder Raumplanungsstudiums gehört auch das Angebot eines einführenden Moduls 1 „Einführung in die Raumplanung (Was ist Raumplanung, Einführung ins Studium)“ im Bachelor, das ebenso auf die Förderung sozialer Kontakte ausgerichtet ist. Der Master Abschluss wird zudem ausdrücklich als Voraussetzung für Promotion und städtebauliches Referendariat dargestellt, was in dieser Prägnanz in keinem der anderen Studiengänge formuliert ist.

\section{Zusammenfassung und Ausblick}

Zusammenfassend lassen sich aus diesem ersten Quervergleich der neuen gestuften Raumplanungsstudiengänge in Deutschland folgende Schlussfolgerungen ziehen:

- Gender Mainstreaming ist keine Zielrichtung der umgesetzten Studienreform. Wenn dies im Einzelfall eingelöst wurde, liegt das nicht an entsprechenden Vorgaben, sondern an vorhandenen Traditionen.

- Die einzelnen Traditionen im Hinblick auf die Integration von Erkenntnissen der Frauen- und Geschlechterforschung werden verstärkt; wo diese nicht vorhanden sind, werden sie auch nicht eingeführt.

- Formelle Unzulänglichkeiten im Bezug auf die sprachliche Differenzierung sind Ausdruck der an den einzelnen Standorten vorherrschenden Werte und Grundhaltungen.

Am bedenklichsten erscheint jedoch die Diskrepanz, die sich zwischen den Welten von Wissenschaft und Praxis auftut: Stadt- und Regional- bzw. Raumplanung bleiben in der Ausbildung bzw. in den neuen Studien- gängen hinter den Anforderungen der Praxis zurück. Die Rolle der Hochschulen als Vorreiterinnen neuer und innovativer Planungskulturen wird nur unzureichend wahrgenommen:

- Während Planungsverwaltungen wie Förderinstitutionen heute angehalten sind, das Prinzip des Gender Mainstreaming in Maßnahmen und Entscheidungen umzusetzen, bereiten die neuen Studiengänge zu wenig auf diese Anforderungen vor. Dies erscheint gerade angesichts der Herausforderungen, die aufgrund des demographischen Wandels und der entstehenden Wissensgesellschaft an Städte und Regionen gestellt werden, fatal.

- Entsprechende Planungsthemen scheinen heute zum Teil schon mehr durch innovative und aufgeschlossene Persönlichkeiten in der Praxis generiert zu werden als an den Hochschulen. Dies ist gerade in Bezug auf die wachsenden Anforderungen an Forschungstätigkeit und Drittmitteleinwerbung auch seitens der Planungsfakultäten kaum vertretbar.

Nicht nur aufgrund allgemeiner Gleichstellungsbestrebungen, sondern auch angesichts der strukturellen Veränderungen, die sich aufgrund des demographischen Wandels im Aufbau der Bevölkerung vollziehen, werden die Anforderungen an Vereinbarkeitsfragen, nicht nur aufgrund der Zunahme bildungshomogener Partnerschaften, künftig steigen. Dass somit auch veränderte Anforderungen auf die Raumplanung zukommen, liegt auf der Hand. Gleichstellungsorientierung kann nicht länger alleinige Aufgabe entsprechend Beauftragter sein, sondern gehört als Querschnittsaufgabe in jedes politische und planerische Themen- und Handlungsfeld. Gerade die Raumplanung, deren Aufgabe es ist, die baulich-räumlichen Voraussetzungen für die $\mathrm{Zu}$ kunft der Gesellschaft zu schaffen, ist ein Feld, in dem auf vernetztes Denken und Handeln nicht verzichtet werden kann. Dieses sollte bereits ganz unten im Bildungsbereich anfangen und sich selbstverständlich an den Hochschulen fortsetzen.

Es wäre im Sinne einer zukunftsfähigen Raumentwicklung daher mehr als angezeigt, dass die verschiedenen Studiengänge ihre Profile in den nächsten Jahren entsprechend anpassen. Dies könnte gleichzeitig ein Beitrag sein zur Vorbeugung gegenüber einem ernsthaften Verlust der Ausbildungen an Praxisrelevanz. 


\section{Anmerkungen}

(1) Bei dem vorliegenden Beitrag handelt es sich nicht um eine systematische Würdigung aller Raumplanungsstudiengänge in Deutschland. Vielmehr sind die der Sichtung zugrunde liegenden Materialien der Autorin von der Schriftleitung dieser Zeitschrift eigens für diesen Artikel zur Verfügung gestellt worden. Es handelt sich dabei um Materialien, die zur Erstellung anderer Beiträge in diesem Heft den jeweiligen Autoren von den angefragten Hochschulen selbst ausgehändigt wurden. Eine systematische Würdigung war aus diesem Grunde nicht möglich und in diesem Rahmen auch nicht vorgesehen. Es ist ein erster Aufriss zum Thema, dem weitere systematische Auswertungen folgen sollten, dies jedoch eher zu einem späteren Zeitpunkt, wenn zu den neuen Studiengängen auch bereits Erfahrungswerte vorliegen.

(2)

$\mathrm{Zu}$ den einschlägigen Studiengängen in Kassel konnten im Rahmen dieses Kurzbeitrags keine Informationen eingeholt werden.

(3)

Dasselbe gilt inzwischen auch für das deutsche Städtebaurecht: So sind gem. Baugesetzbuch (BauGB) in der aktuellen Fassung vom 21.12.2006 gem. $\$ 1$ Abs. 6 Nr. 3 bei der Aufstellung der Bauleitpläne die unterschiedlichen Auswirkungen von Planungen auf Frauen und Männer zu berücksichtigen.

(4)

Vgl. www.bolognaprozess-gender.uni-siegen.de/

(5)

$\$ 6$ Abs. 2 Gesetz zur Errichtung einer Stiftung „Stiftung zur Akkreditierung von Studiengängen in Deutschland" vom 15.2.2005

(6)

Inwiefern die Studienkommissionen an den entsprechenden Fakultäten pro Mitgliedergruppe zu gleichen Teilen aus Frauen und Männern zusammengesetzt waren, wurde im Rahmen dieses Beitrags nicht untersucht.

(7)

Dieser Prüfung lagen die Unterlagen zugrunde, die der Autorin für diesen Zweck von den Schriftleitern dieser Zeitschrift zur Verfügung gestellt wurden:

- Uni Dortmund: Raumplanung, Modulhandbücher BSc und MSc vom 29.9.2007; Internetpräsentation und Infobroschüre zum BSc und zu den Vertiefungsrichtungen im MSc; Fleischhauer/Greiwe/Scholz (2007).

- TU Kaiserslautern: Bachelor of Science Raumplanung, Modulhandbuch Stand: 06/2007.

- TU Berlin: Stadt- und Regionalplanung, Studienordnungen für den Bachelor- und für den Masterstudiengang vom 14.2. und 12.7.2006.

- HCU Hamburg: Stadtplanung und Stadtentwicklung, Information für Studienbewerber (Bachelor: Stadtplanung, Master: Stadtplanung und Stadtentwicklung) in deutsch und eng. lisch mit Bachelor-PO, exemplarischer Studienplan, Stand: $10 / 2005$

- BTU Cottbus: Stadt- und Regionalplanung, allgemeine BaMa Informationen o. J., zusätzlich aus dem Netz geholt: Prüfungsund Studienordnungen für den Bachelor- und für den Masterstudiengang vom 2.6.2005.

Unterlagen über die Studiengänge in Stadt- und Regionalplanung an der Universität Kassel fehlten; inwieweit das Pflichtmodul im hiesigen BA „Gesellschaft und Umwelt" Genderinhalte umfasst, konnte ohne zusätzliche Recherchen nicht festgestellt werden. Solche waren in diesem Rahmen nicht möglich.
(8)

Dies dürfte insbesondere auf die entsprechende fachliche Tradition an diesen Studienstandorten sowie auf eine entsprechende Sensibilisierung (von maßgeblichen Teilen) des Lehrkörpers zurück zu führen sein.

(9) Immerhin werden hier seit einigen Jahren für alle Fakultäten regelmäßig Weiterbildungsveranstaltungen zum Gender Mainstreaming in Lehr- und Studieninhalten angeboten.

\section{Literatur}

ASAP - Akkreditierungsverbund für Studiengänge der Architektur und Planung (Hrsg.) (2004): Fachliche Standards für die Akkreditierung von Studiengängen der Stadtplanung/Raumplanung. Studiengänge Stadtplanung/Regionalplanung, Landesplanung und Raumplanung. Berlin (3. Aufl. 2008).

Becker, R.; Jansen-Schulz, B.; Kortendiek, B.; Schäfer, G. (2006): Gender-Aspekte bei der Einführung und Akkreditierung gestufter Studiengänge - eine Handreichung. Dortmund.

Bührmann, A.D. (2006): Gender Studies im Bologna-Prozess. Herausforderung oder Chance? Vortrag beim Jahreskongress 2006 der Arbeitsgemeinschaft für Hochschuldidaktik (AHD) vom 6. bis 9.3.2006 in Dortmund. Stand: März 2006. http://www.hd-online. de/ahdkongress06/fileadmin/presentation/panel2/Andrea. Buehrmann.pdf

Dörhöfer, K.; Flecken, U.; Zibell, B. (1998): Frauenforschung und Gender Studies in der Stadt- und Regionalplanung. In: Bohne, R.; Jost, F; Mack, G.; Schütze, B. (Hrsg.): Stadtplanung und Städtebau. Positionen finden - Überzeugungen vermitteln. Eine Festschrift für Dieter Frick. Berlin, S. 43-57. = SRL-Schriftenreihe, Bd. 43.

Fleischhauer, T.; Greiwe, U.; Scholz, T. (2007): BSc/MSc-Studiengänge der Raumplanung in Dortmund. In: RaumPlanung H. 132/33, S. 174-176.

Konferenz der Europäischen Hochschulministerinnen und -minister (2003): Realising the European Higher Education Area. Stand: September 2003. www.bologna-berlin2003.de/pdf/ Communique1.pdf

Kultusministerkonferenz (2004): Eckpunkte für die Weiterentwicklung der Akkreditierung in Deutschland. Beschluss der Kultusministerkonferenz vom 15.10.2004.

Löther, A. (Hrsg.) (2004): Europäisierung der Gleichstellung Bologna-Prozess - Hochschulstrukturen - Forschungspolitik. Dokumentation der 15. Jahrestagung der Bundeskonferenz der Frauenbeauftragten und Gleichstellungsbeauftragten an Hochschulen. Bonn.

Prof. Dr. Barbara Zibell

Leibniz Universität Hannover

Fakultät Architektur und Landschaft

Institut für Geschichte und Theorie

Abt. Planungs- und Architektursoziologie

Herrenhäuser Straße 8

30419 Hannover

E-Mail: b.zibell@igt-arch.uni-hannover.de 